\title{
Comparative Study of Live Virtual Machine Migration Techniques in Cloud
}

\author{
Gulshan Soni \\ M.E. Student, \\ Department of Computer Science \\ NITTTR, Chandigarh
}

\author{
Mala Kalra \\ Assistant Professor \\ Department of Computer Science \\ NITTTR, Chandigarh
}

\begin{abstract}
Virtualization technology is currently becoming increasingly popular and valuable in cloud computing environments due to the benefits of server consolidation, live migration, and resource isolation. Live virtual machine migration is the process of moving virtual machine from one physical host to other without disturbing the users. Effective migration of virtual machine requires the movement of storage, memory, process states and network connectivity. Live migration of virtual machines has been a powerful tool to facilitate system maintenance, load balancing, fault tolerance, and powersaving, especially in clusters or data centers. During the process of migration, services are running in the virtual machines, hence it requires minimum total migration time, down time in order to avoid service degradation. This paper introduced discussion of various techniques for effective live virtual machine migration to reduce both downtime and total migration time. This paper also present the various scenarios, where the live migration of virtual machines can takes place.
\end{abstract}

\section{Keywords}

Virtual Machine (VM), Live Migration, Pre-copy, Post-copy, Virtualization.

\section{INTRODUCTION}

Cloud computing means applications and services offered through the Internet. These services are accessible from data centers to all over the world, which jointly are referred to as the "cloud". The idea of the "cloud" simplifies many network connections and computer systems involved in online services. The essential characteristics of cloud computing are rapid elasticity, on-demand self-service, resource pooling, broad network access, and measured service. Cloud computing services describe the type of service that the cloud service provider is offering. Cloud computing offers mainly three type of services such as Software as a Service (SaaS), Platform as a Service (PaaS) and Hardware as a Service (HaaS). Cloud Computing Deployment Models refer to the location and management of the infrastructure cloud services. The Deployment Model of cloud computing are Private Cloud, Community cloud, Public cloud and Hybrid cloud. The key technology behind cloud computing is virtualization. Virtualization has enabled resource pooling without which it is impossible to attain efficient utilization and provide reasonable cost to users. Virtualization has provided a means for migration of virtual machines from one server to another. Live migration of virtual machine is a process of moving a running virtual machine from one physical machine to another physical machine without disconnecting the client or application. The benefits of live virtual machine migration are energy management, load balancing and fault tolerance.

This paper will discuss about various Live Virtual Machine Migration techniques. This paper is organized as following:
Section 2 gives brief introduction about virtualization technology. Section 3 describes about Virtual machine migration and its types. Live virtual machine migration techniques are surveyed in section 4 . Section 5 describes about various scenarios where live virtual machine migration is needed. Conclusion of work is presented in section 6 .

\section{VIRTUALIZATION}

Virtualization is a method for creating multiple virtual machines that run on a single physical machine. Virtualization allows for a smaller number of high-powered servers to create a larger number of less-powered servers while reducing the overall cost in space, power, and other infrastructure.

Figure 1 describes the basic concept of virtualization that consist of physical machine (PM), Virtual Machine Monitoring (VMM) and Virtual Machines (VMs).

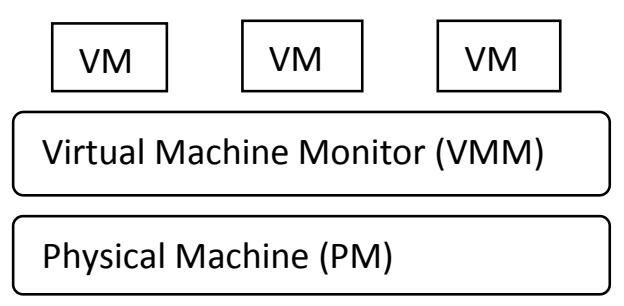

Figure 1.Virtulization

The real advantage of virtualized servers and hardware are that if one virtual machine is at or near idle, others present in same physical machine can take advantage of its resources to perform their processing. This is the same in principle as clustered groups of servers, but virtualization allows for an even higher level of resource sharing.

The way a virtualized server functions is that it introduces an abstraction layer between the hardware and the operating system. This abstraction layer is called the hypervisor.

The characteristics of virtual machine include

1. Isolation.

2. Ease of testing.

3. Mobility.

\section{Hypervisor are divided into two types}

\subsection{Bare Metal Hypervisor}

Bare Metal Hypervisor is installed on the hardware itself. The Bare metal hypervisor has a significantly higher level of control over the resources available to it because it does not have to go through an intermediary to access the resources. Examples: VMware, HyperV 
Figure 2 describes the working of Bare Metal Hypervisor.

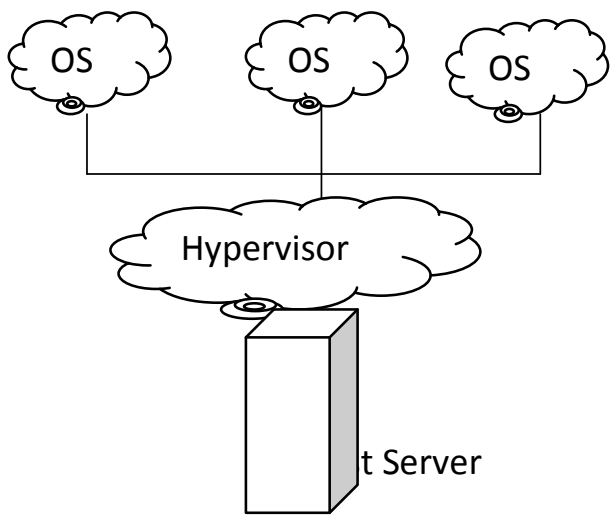

Figure 2. Bare Metal Hypervisor

\subsection{Hosted Hypervisor}

Hosted Hypervisor is not installed on the hardware itself, but within an operating system on the server, and thus is under the control of that operating system. Hosted hypervisors are significantly easier to access, set up, and manage as they are normally able to take advantage of the parent operating systems graphical user interface to provide their own graphical management system.

Example: VMware Player, VirtualBox, Xen

Figure 3 describes the working of Hosted Hypervisor.

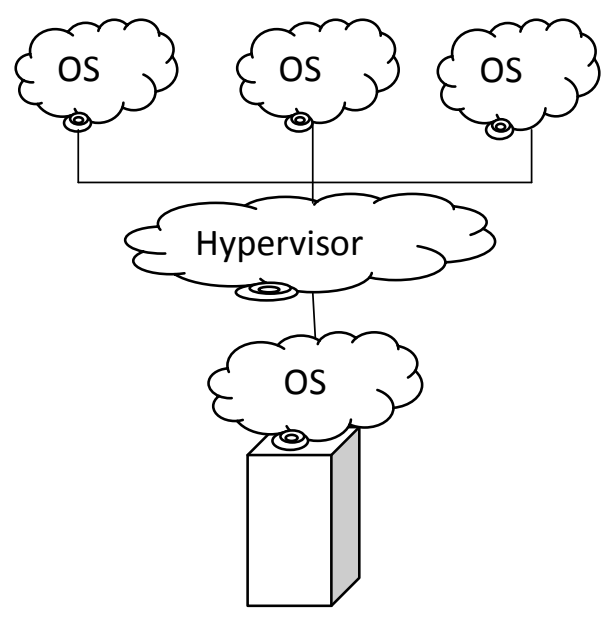

Host Server

Figure 3. Hosted Hypervisor

\section{VIRTUAL MACHINE MIGRATION}

Virtual machine migration is the process of moving virtual machine from one physical host to other host without disturbing others. Migrating an entire OS and all of its applications as one unit between physical machines.

Migration of VM can be divided into two categories:

a) Off-line VM Migration: Off-line VM migration process pause the VM and transfer all the states of VM to target host then finally resume the VM in the new host. The advantage is simple procedure and disadvantage is long downtime. b) Live VM Migration: In live VM migration state of VM is transferred with minimum service disruption from one host to other host. The Key advantage of live VM migration is user-invisible downtime with fast network.

Figure 4 describes live virtual machine migration. Three physical Machines with virtualization layer host five instances of operating systems (with one being migrated between PM 2 and PM 3).

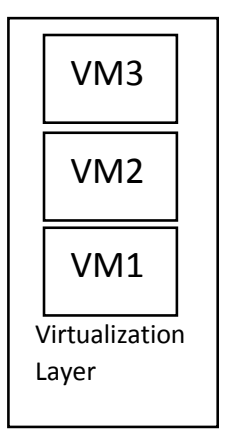

PM1

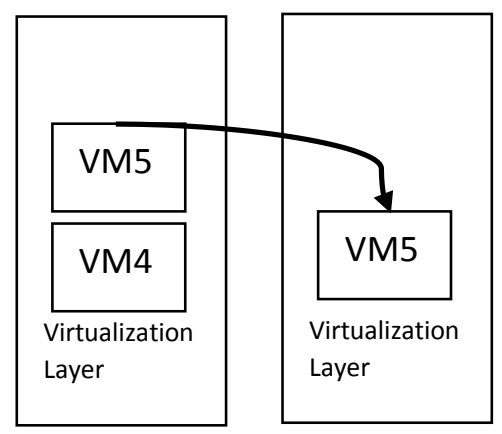

PM2

PM3
Figure 4: Virtualized servers with live migration capability

Live virtual machine migration having mainly following performance metrics.

1. Total migration time: It is defined as the total time taken to migrate a virtual machine from its host machine to the target machine.

2. Down time: Down time is defined as the duration of time for which services are not available to the users.

3. Amount of migrated data: During the Live migration how much data is transferred form one host to another host.

4. Migration overhead: Migration overhead consist of system resource consumption.

5. Application degradation: The extent to which migration slows down the applications executing within the migrating VM.

\section{LIVE VM MIGRATION TECHNIQUES}

The first technique of migration of VMs was stop-and-copy approach [1], which involved halting the targeted VM, copying all pages to the destination, and then start execution of that VM. The advantage of stop-and-copy approach is simplicity but the service downtime is more in this approach. In the stopand-copy approach service downtime is proportional to the amount of physical memory of VM. These types of approaches are not acceptable in live running scenarios. The techniques, which are used for live migration of virtual machines are discussed below:

\subsection{Pre-copy Approach}

In a pre-copy approach all states of hardware and software running within a VM are encapsulated. Which migrating a VM three kind of states are required that are virtual device states, external connections and physical memory of VM. This approach begins with transferring the memory contents to the target machine. After completing the memory transfer other states such as processor states etc. are transferred to destination.

The actual migration process involves following steps [2]: 
1. Initiating the migration by selecting the VM to migrate and its destination.

2. Pre-copying the memory state of the VM to the destination while the VM is running on the source.

3. Quiescing the VM and sending the non-memory state.

4. Transferring control of the VM to the destination and resuming it at the destination.

5. Sending any remaining memory state and removing the dependency on the source machine.

The basic pre-copy approach is suggested by Michael Nelson et al. [2]. They proposed first system that provided transparent virtual machine migration of existing applications and operating systems, where neither the applications nor the operating systems need to be modified. It is the first research to provide performance measurements of hundreds of virtual machine migrations of concurrently running virtual machines with standard industry benchmarks. It characterizes the overheads and resources required during virtual machine migration. They described a migration system, named VMotion that has an integral part of the VMware Virtual Center product.

In data centers and clusters environment migrating operating systems (OSes) instances across different physical hosts is a useful tool for administrators. Clark et.al.[3] deliberated the design options for migrating OSes running services with liveness constraints, focusing on data center and cluster environments. They introduced and analyzed the concept of writable working set, and presented the design, implementation and evaluation of high performance OS migration built on top of the Xen VMM.

\section{Improvement over Pre-copy Approach:}

In order to minimize the downtime of migration of virtual machine several authors have proposed improvement over the basic pre-copy approach.

Live migration of virtual machine's downtime directly depends on the size of memory state of the VM that is going to migrate from one host to another host. To minimize the size of memory state of the VM different memory compression techniques are proposed.Hai Jin et al. [4], presented the design and implementation of a novel memory compression based VM migration approach (MECOM) that first uses memory compression to provide fast, stable virtual machine migration, while guaranteeing the virtual machine services to be slightly affected. They design an adaptive zero aware compression algorithm for balancing the performance and the cost of virtual machine migration. Pages are quickly compressed in batches on the source and exactly recovered on the target.

Another memory compression technique is proposed by Svard et al.[5]. They implemented the application of delta compression during the transfer of memory pages in order to increase migration throughput and thus reduce downtime. The delta compression live migration algorithm is implemented as a modification to the KVM hypervisor. Its performance is evaluated by migrating VMs running different type of workloads and the evaluation demonstrates a significant decrease in migration downtime in test cases. An enterprise application scenario, the delta compression algorithm successfully live migrates a very large system that fails after migration using the standard algorithm. Finally, they discussed some general effects of delta compression on live migration and analysed when it is beneficial to use this technique.
There are some other techniques which are useful in minimizing the downtime of live migration of virtual machines. While transfer of memory state of the VM marked the frequently updated pages and transfer these dirty pages at last iteration of memory transfer. Fei Ma et al. [6], added a bitmap page in his improved pre-copy approach, which marks those frequently updated pages. By the judge in the iteration process, it put those frequently updated pages into the page bitmap, and those pages can only be transmitted in the last round of the iteration process. This can ensure that those frequently updated pages are transmitted just once in the iteration process.

Bolin Hu et al. [7] also proposed an improved time-series based pre-copy approach for virtual machine migration. With the time-series prediction technique, they identified frequently updated dirty pages (high dirty pages) in the past and future period more precisely, and transmit them in the last round of iteration, in order to reduce unnecessary, repeated transmission of dirty pages.

Ibrahim et al. [8] presented a novel algorithm that achieves both low downtime and low application performance impact. At the core of the algorithm is detecting memory update patterns and terminating migration when improvements in downtime are unlikely to occur. They implemented this approach in KVM and demonstrated its benefits for both Ethernet and RDMA (InfiniBand) migration.

\subsection{Post-copy Approach}

The Post-copy approach is proposed by Michael et al [9]. The Post-Copy approach is opposite to pre-copy approach for live migration of VMs.In the post-copy approach, first it migrates the VM's execution states such as CPU states etc. and after migration of CPU state, it transfers VM's memory pages. The main benefit of this approach is that each memory page is transferred at most once, thus avoiding the duplicate transmission overhead of pre-copy.

Michael et al [9], presented the design, implementation, and evaluation of post-copy based live migration for virtual machines (VMs) across a Gigabit LAN. The post-copy strategy can provide a "win-win" by reducing total migration time while maintaining the liveness of the VM during migration. They facilitated the use of post-copy with adaptive pre paging techniques to minimize the number of page faults across the network. Finally, they eliminate the transfer of free memory pages in both pre-copy and post-copy through a dynamic selfballooning (DSB) mechanism. DSB periodically reclaims free pages from a VM and significantly speeds up migration with negligible performance impact on VM workload.

\subsection{Other Approaches}

Many previous approaches focused on transferring memory but there are some techniques which used different approaches to provide fast transparent live migration of virtual machines. Weining Lie [10], described a novel approach based on recovering system and CPU scheduling to provide fast, transparent live migration. Target host executes log files generated on source host to synchronize the states of source and target hosts, during which a CPU scheduling mechanism is used to adjust the log generation rate.

Hai kum liu [11], described the design and implementation of a novel approach CR/TR-Motion that adopts Check pointing/recovery and trace/replay technology to provide fast, 
transparent VM migration. With execution trace logged on the source host, a synchronization algorithm is performed to orchestrate the running source and target VM until they get a consistent state. This scheme can greatly reduce the migration downtime and network bandwidth consumption.

\section{Live virtual machines migration for cloud federation:}

In current scenario cloud service provider does not offer interoperability, which is interruption to the advancement and adoption of the cloud computing paradigm.In the cloud federation, movement of VMs from a server of one cloud provider to a server of another cloud provider. The future of the cloud computing is cloud federation because it provide interoperability among cloud service providers.

Elmroth et al. [12] proposed technology neutral interfaces and architectural additions for handling placement, migration, and monitoring of VMs in federated cloud environments, the latter as an extension of current monitoring architectures used in Grid computing. The interfaces presented adhere to the general requirements of scalability, efficiency, and security in addition to specific requirements related to the particular issues of interoperability and business relationships between competing cloud computing infrastructure providers.

Antonio et al. [13] reduced consumption of bandwidth and cloud resources costs proposing a Composed Image Cloning (CIC) methodology. This approach does not consider the diskimage of a VM as a single monolithic block, but as a combination between "composable" and "user data" blocks. Since the former may be cloned locally in the destination clouds, the amount of data which have to be transferred may be reduced.

Chun-Hui Suen [14], proposed and evaluated techniques for efficient and effective transfer and storage of VM images, which have high duplication, for both instance and volumebased cloud storage. They focused is on both the public and private cloud infrastructure and the movement of VMs between them. In this paper, different techniques for both instancebased and volume-based storage in the public and private cloud infrastructure were proposed and compared.

\section{LIVE MIGRATION IN DIFFERENT SCENARIOS}

Live virtual machine migration is used in the following scenarios of cloud computing:

\subsection{Power Management}

It means lowering the energy usage of data centers. Computing applications and data are growing so quickly that increasingly larger servers and disks are needed to process them fast enough within the required time period.

Anton et al. [15] defined an architectural framework and principles for energy-efficient Cloud computing. This proposed energy-aware allocation heuristics provision data center resources to client applications in a way that improves energy efficiency of the data center, while delivering the negotiated Quality of Service (QoS).

Jeffrrey et al. [16] presented the design and implementation of an architecture for resource management in a hosting center operating system, with an emphasis on energy as a driving resource management issue for large server clusters. The goals are to provision server resources for co-hosted services in a way that automatically adapts to offered load, improve the energy efficiency of server clusters by dynamically resizing the active server set, and respond to power supply disruptions or thermal events by degrading service in accordance with negotiated Service Level Agreements (SLAs).

\subsection{Proactive Maintenance}

Proactive maintenance deals with replacing the failure virtual machine by moving in to new physical machine.

Arun et al. [17] promoted a proactive technique where processes automatically migrate from "unhealthy" nodes to healthy ones. This approach relies on operating system virtualization techniques exemplified by but not limited to Xen. This paper contributes an automatic and transparent mechanism for proactive FT for arbitrary MPI applications.

Troger et al. [18] proposed an architectural blueprint for managing server system dependability in a pro-active fashion, in order to keep service-level promises for response times and availability even with increasing hardware failure rates. They introduced the concept of anticipatory virtual machine migration that proactively moves computation away from faulty or suspicious machines. The migration decision is based on health indicators at various system levels that are combined into a global probabilistic reliability measure.

\subsection{Load Balancing}

Load balancing is the process of sharing the load among computers in order to optimize the utilization of available CPU resources.

A number of techniques proposed for load balancing are based on live virtual machine migration. Ma et al. [19] proposed a new model for distributed load balancing allocation of virtual machine in cloud data center using the TOPSIS method which is one of the most efficient Multi Criteria Decision Making (MCDM) technique. This method can find the most suitable physical machine in the data center for the migrated VMs. MCDM technique try to avoid the live virtual machine migration.

Zhao et al. [20] proposed a distributed load balancing algorithm COMPARE_AND_BALANCE based on sampling to reach an equilibrium solution. They designed and implemented a simple model which decreases the migration time of virtual machines by shared storage and fulfills the zerodowntime relocation.

\section{COMPARATIVE STUDY OF DIFFERENT LIVE VM MIGRATION TECHNIQUES}

In this section will describe compare various techniques used for live migration of VMs with their basic concept and their pros and cons. This paper discussed about different approaches that are used for live migration of virtual machines. Each approach consists of some advantages and disadvantages.

The two main techniques that are used for live migration of virtual machines are pre-copy and post-copy techniques. The basic concept of pre-copy is very simple that is if one VM want to migrate form one physical host to another physical host than first transfer their device states, network connections and physical memory to remote location and finally transfer the processors states to that location. During the migration, the client connection is maintained and client feel little service 
downtime .The post-copy techniques are just opposite of precopy techniques. In post copy technique, firstly the processor states are transferred than the physical memory etc. transfer. A lot of techniques for the improvement of pre-copy approach have been proposed. The improvement over pre-copy is based on how can reduce the amount of physical memory transfer from one physical machine to other physical machines. Many memory compression techniques and frequently updated pages of memory techniques are used to reduce the size of physical memory of virtual machine.

Table 1. Comparison of different live migration of virtual machines techniques

\begin{tabular}{|c|c|c|c|c|c|}
\hline \multicolumn{3}{|c|}{ Virtual Machine Migration Techniques } & Concept & Pros & Cons \\
\hline \multicolumn{3}{|c|}{ Stop-and-Copy approach [1] } & $\begin{array}{l}\text { Halting the targeted VM, } \\
\text { copying all pages to the } \\
\text { destination, and then start } \\
\text { execution of that VM. }\end{array}$ & 1. Simplicity & $\begin{array}{l}\text { 1. More Service } \\
\text { downtime }\end{array}$ \\
\hline \multicolumn{3}{|c|}{ Pre-Copy approach [2] } & $\begin{array}{l}\text { Transfer VM's memory } \\
\text { contents first, then processor } \\
\text { states are transferred to } \\
\text { destination. }\end{array}$ & $\begin{array}{l}\text { 1. Application downtime caused } \\
\text { by migration is less than a } \\
\text { second. } \\
\text { 2. Migration is completely } \\
\text { transparent to the application, } \\
\text { the operating system and } \\
\text { remote clients. }\end{array}$ & $\begin{array}{l}\text { 1. } \begin{array}{l}\text { Duplicate } \\
\text { transmission } \\
\text { overhead }\end{array} \\
\end{array}$ \\
\hline \multicolumn{3}{|c|}{ Adaptive Rate Limiting approach[3] } & $\begin{array}{l}\text { Used the concept of writable } \\
\text { working set and showed OS } \\
\text { migration built on top of the } \\
\text { Xen VMM. }\end{array}$ & $\begin{array}{l}\text { 1. Service downtimes as low as } \\
60 \mathrm{~ms} \text {. }\end{array}$ & $\begin{array}{l}\text { 1. Large total } \\
\text { migration time }\end{array}$ \\
\hline \multirow{5}{*}{$\begin{array}{l}\text { Improved } \\
\text { Pre-Copy } \\
\text { approach }\end{array}$} & \multirow{2}{*}{$\begin{array}{l}\text { Memory } \\
\text { Compression } \\
\text { Techniques }\end{array}$} & $\begin{array}{l}\text { adaptive } \\
\text { memory } \\
\text { compressi } \\
\text { on } \\
\text { approach } \\
\text { [4] }\end{array}$ & $\begin{array}{l}\text { Memory compression based } \\
\text { VM migration approach } \\
\text { (MECOM) that first uses } \\
\text { memory compression. }\end{array}$ & $\begin{array}{l}\text { 1. It provides fast and stable } \\
\text { virtual machine migration. } \\
\text { 2. Minimizes the downtime and } \\
\text { total migration time. }\end{array}$ & $\begin{array}{l}\text { 1. Compression } \\
\text { operations } \\
\text { introduced } \\
\text { additional } \\
\text { overhead. }\end{array}$ \\
\hline & & $\begin{array}{l}\text { delta } \\
\text { compressi } \\
\text { on }[5]\end{array}$ & $\begin{array}{l}\text { The delta compression live } \\
\text { migration algorithm is } \\
\text { implemented as a } \\
\text { modification to the KVM } \\
\text { hypervisor }\end{array}$ & $\begin{array}{l}\text { 1. Downtime is reduced. } \\
\text { 2. Increases migration } \\
\text { throughput. }\end{array}$ & $\begin{array}{l}\text { Compression } \\
\text { operations } \\
\text { introduced } \\
\text { additional } \\
\text { overhead. }\end{array}$ \\
\hline & \multirow{3}{*}{$\begin{array}{l}\text { Frequently } \\
\text { updated pages } \\
\text { of memory. }\end{array}$} & $\begin{array}{l}\text { page } \\
\text { bitmap } \\
{[6]}\end{array}$ & $\begin{array}{l}\text { By the judge in the iteration } \\
\text { process, it put those } \\
\text { frequently updated pages } \\
\text { into the page bitmap, and } \\
\text { those pages can only be } \\
\text { transmitted in the last round } \\
\text { of the iteration process }\end{array}$ & $\begin{array}{l}\text { It reduces } 34 \% \text { of total } \\
\text { transferred data and } 32.5 \% \text { of } \\
\text { total migration time. }\end{array}$ & $\begin{array}{l}\text { 1. Not suitable } \\
\text { for wide-area } \\
\text { live virtual } \\
\text { machine } \\
\text { migration. }\end{array}$ \\
\hline & & $\begin{array}{l}\text { time- } \\
\text { series } \\
\text { predictio } \\
\text { n } \\
\text { technique } \\
\text { [7] }\end{array}$ & $\begin{array}{l}\text { With the time-series } \\
\text { prediction technique, it } \\
\text { identified frequently updated } \\
\text { dirty pages (high dirty } \\
\text { pages) in the past and future } \\
\text { period more precisely, and } \\
\text { transmit them in the last } \\
\text { round of iteration }\end{array}$ & $\begin{array}{l}\text { 1. Less number of iterations } \\
\text { 2. Less down time and } \\
\text { migration time. } \\
\text { 3. Fewer pages transferred }\end{array}$ & $\begin{array}{l}\text { 1. This approach } \\
\text { is useful when } \\
\text { high dirty } \\
\text { pages in } \\
\text { iteration. }\end{array}$ \\
\hline & & $\begin{array}{l}\text { detecting } \\
\text { memory } \\
\text { update } \\
\text { patterns } \\
{[8]}\end{array}$ & $\begin{array}{l}\text { Detecting memory update } \\
\text { patterns and terminating } \\
\text { migration. }\end{array}$ & $\begin{array}{l}\text { Provide minimal downtime } \\
\text { and minimal impact on end- } \\
\text { to-end application } \\
\text { performance. }\end{array}$ & $\begin{array}{l}\text { 1. Useful for } \\
\text { memory } \\
\text { intensive } \\
\text { applications. }\end{array}$ \\
\hline
\end{tabular}




\begin{tabular}{|c|c|c|c|c|}
\hline \multicolumn{2}{|c|}{ Post-copy Approach [9] } & $\begin{array}{l}\text { First it migrates the VM's } \\
\text { execution states than all } \\
\text { memory pages are } \\
\text { transferred only once during } \\
\text { the whole migration process }\end{array}$ & $\begin{array}{l}\text { 1. Each memory page is } \\
\text { transferred at most once. } \\
\text { 2. Baseline total migration time } \\
\text { is achieved. }\end{array}$ & $\begin{array}{l}\text { 1. Downtime is } \\
\text { much higher } \\
\text { than that of pre } \\
\text { copy. }\end{array}$ \\
\hline \multirow[b]{2}{*}{$\begin{array}{l}\text { Other } \\
\text { Approaches }\end{array}$} & $\begin{array}{l}\text { Recovering system } \\
\text { CPU scheduling [10] }\end{array}$ & $\begin{array}{l}\text { A novel approach based on } \\
\text { recovering system and CPU } \\
\text { scheduling. }\end{array}$ & $\begin{array}{l}\text { 1. It can reduce the downtime } \\
\text { and total migration time in } \\
\text { compares with pre-copy } \\
\text { algorithm. }\end{array}$ & $\begin{array}{l}\text { 1. It is not sure } \\
\text { this approach } \\
\text { can work well } \\
\text { in more } \\
\text { complex } \\
\text { environment. }\end{array}$ \\
\hline & $\begin{array}{l}\text { Check pointing/recovery } \\
\text { and trace/replay approach } \\
\text { (CR/TR-Motion) }[11]\end{array}$ & $\begin{array}{l}\text { Transfers execution trace } \\
\text { file in iterations rather than } \\
\text { dirty pages, which is logged } \\
\text { by a trace daemon. } \\
\text { Apparently, the total size of } \\
\text { all log files is much less than } \\
\text { that of dirty pages. }\end{array}$ & $\begin{array}{l}\text { 1. Provides fast and transparent } \\
\text { VM migration. } \\
\text { 2. Total migration time and } \\
\text { downtime of migration are } \\
\text { drastically reduced }\end{array}$ & $\begin{array}{l}\text { 1. CR/TR Motion } \\
\text { is valid only } \\
\text { when the log } \\
\text { replay rate is } \\
\text { larger than the } \\
\text { log growth rate }\end{array}$ \\
\hline
\end{tabular}

\section{CONCLUSION}

This paper presents a review of various live virtual machine migration techniques in cloud computing. The live virtual machine migration techniques can be broadly divided into two categories that is pre-copy and post-copy approach. Few techniques proposed by researchers other than these two approaches are also discussed. The paper also discussed VM migration techniques for cloud federation. All the techniques discussed above try to minimize the total downtime of migration and provide better performance in low bandwidth. Then the various scenarios are presented where the migration of virtual machine are needed. The comparative study has been made on different live virtual machine migration techniques with their pros and cons.

\section{REFERENCES}

[1] C. P. Sapuntzakis, R. Chandra, B. Pfaff, J. Chow, M. S. Lam, and M. Rosenblum 2002. Optimizing the migration of virtual computers. Proceedings of the 5th Symposium on Operating Systems Design and Implementation (OSDI-02).

[2] M. Nelson, B. Lim, and G. Hutchines 2005. Fast transparent migration for virtual machines. Proceedings of the USENIX Annual Technical Conference (USENIX'05), 391-394.

[3] C. Christopher, F. Keir, H. Steven, H. Jacob Gorm, J. Eric, L. Christian, P. Ian, and W. Andrew 2005. Live migration of virtual machines. 2nd conference on Symposium on Networked Systems Design \& Implementation - Volume 2: USENIX Association.

[4] H. Jin, L. Deng, S. Wu, X. H. Shi, and X. D. Pan 2009. Live Virtual Machine Migration with Adaptive Memory Compression. IEEE International Conference on Cluster Computing, 1-10.

[5] P. Svard, J. Tordsson, B. Hudzia, and E. Elmroth 2011. High performance live migration through dynamic page transfer reordering and compression. 3rd IEEE International Conference on Cloud Computing Technology and Science, CloudCom, 542-548.
[6] F. Ma, F. Liu, and Z. Liu 2010. Live Virtual Machine Migration Based on Improved Precopy Approach. Proceedings Software Engineering and Service Sciences, 230-233.

[7] B.Hu, Z. Lei, Y. Lei, D. Xu and J. Li 2011. A TimeSeries Based Precopy Approach for Live Migration of Virtual Machine. IEEE 17th International Conference on Parallel and Distributed Systems, 947-952.

[8] K. Z. Ibrahim, S. Hofmeyr, C. Iancu, and E. Roman 2011. Optimized precopy live migration for memory intensive applications. International Conference for High Performance Computing, Networking, Storage and Analysis (SC), 1-11.

[9] R. H. Michael, D. Umesh, and G. Kartik 2009. Post-copy live migration of virtual machines. ACM Special Interest Group on Operating Systems, 14-26.

[10] L. Weining and F. Tao 2009. Live migration of virtual machine based on recovering system and CPU scheduling. 6th IEEE joint International Information Technology and Artificial Intelligence Conference,Piscataway, NJ, USA, 303-7.

[11] L. Haikun, J. Hai, L. Xiaofei, H. Liting, and Y. Chen, , 2009. Live migration of virtual machine based on full system trace and replay. 18th ACM International Symposium on High performance distributed computing, 101-110.

[12] E. Elmroth and L. Larsson 2009. Interfaces for placement, migration, and monitoring of virtual machines in federated clouds. 8th International Conference on Grid and Cooperative Computing, 253-260.

[13] A. Celesti, F. Tusa, M. Villari, and A. Puliafito, 2010. Improving virtual machine migration in federated cloud environments. 2nd International Conference on Evolving Internet, Internet, 61-67.

[14] S. Chun-Hui, M. Kirchberg, and L. Bu Sung Efficient 2011. Migration of Virtual Machines between Public and Private Cloud. IEEE Third International conference on Cloud Computing Technology and Science (CloudCom), 549-53. 
[15] Anton Beloglazov, Jemal Abawajy, Rajkumar Buyya 2012. Energy aware resource allocation heuristics for efficient management of data centers for cloud computing. Future Generation computer systems, 755768.

[16] Jeffrrey S.Chase, Darrell C Anderson 2001. Managing Energy and Server Resources in Hosting centers. Proceedings of the eighteenth ACM symposium on Operating systems principles (SOSP '01), 103-116.

[17] Arun Babu Nagarajan, Frank Mueller, Christian Engelmann, Stephen L.Scott 2007. Proactive fault tolerance for HPC with Xen Virtualization. Proceedings of the $21 \mathrm{st}$ annual international conference on supercomputing.
[18] Troger P, Salfner F 2011. Timely virtual machine migration for pro-active fault tolerance. 14th IEEE International Symposium.

[19] F. M a, F. Liu and Z. Liu 2012. Distributed Load Balancing Allocation of Virtual Machine in Cloud Data Center. IEEE 3rd International conference on Software Engineering and Service Science (ICSESS), 20-23.

[20] Y. Zhao, and W. Huang 2009. Adaptive Distributed Load Balancing Algorithm based on Live Migration of Virtual Machines in Cloud. Proceedings of 5th IEEE International Joint Conference on INC, IMS and IDC Seoul, Republic of Korea, 170-175. 